\title{
Collaborative Teleradiology
}

\author{
Krzysztof Zieliński ${ }^{1}$, Jacek Cała ${ }^{1}$, Łukasz Czekierda ${ }^{1}$, Sławomir Zieliński ${ }^{1}$ \\ ${ }^{1}$ Dept. of Computer Science, AGH - University of Science and Technology, \\ al. A. Mickiewicza 30, 30-059 Kraków, Poland \\ $\{$ kz,cala,luke,slawek\}@cs.agh.edu.pl \\ http://www.cs.agh.edu.pl
}

\begin{abstract}
The paper introduces a notion of collaborative teleradiology as a novel organisation of information exchange during a remote radiological consultation. A general interaction pattern between medical experts taking part in a collaborative teleradiology session has been defined. This session has been compared to interaction typically performed in WWW centric teleradiology systems. Next technical implication of a proposed information exchange paradigm has been elaborated. Finally two prototype tools TeleNegatoscope and TeleDICOM that supporting of the collaborative teleradiology working environment have been described in more details.
\end{abstract}

\section{Introduction}

Most of the existing teleradiology [1] and PACS systems [2] nowadays are oriented on asynchronous communication between a radiology expert and other medical staff. The applied communication pattern assumes communication via a central database where results of radiology investigations are stored. These radiology data could be accessed either locally or remotely via computer network by radiology experts in order to make diagnoses which are stored back into the database and can be further accessible for authorized medical personnel. This co-operation pattern has been recently enhanced with WWW technology application that provides standardised uniform platform for remote access from any computer attached to a computer network to the data stored in the database. The application of WWW technology does not remove two important characteristic features of the discussed pattern i.e. lack of real-time interactivity between a radiology expert and other doctors, and sensitivity to computer network quality of service (QoS) parameters.

Progress in distributed computer system architectures and software technologies $[3,4,5]$ offers possibility of developing new communication patterns suitable for teleradiology applications which do not suffer from the pointed out deficiencies. These patterns make it possible to achieve on-line communication between experts in a medical reference centre and doctors in a peripheral hospital and collaborative synchronous work on the same radiology data.

This paper introduces a notion of collaborative teleradiology, which assumes that a group of medical experts located in different clinics or hospitals work synchronously on the same radiology data sharing access to them and exchanging comments by using computer network. In that sense it brings a teleworking environment in medicine on higher, more advanced, level. Collaborative teleradiology addresses not 
only telediagnosis but also teleconsultancy issues by providing tools and mechanisms enabling on-line exchange of opinions. The key challenge from the technical point of view is how to implement a collaborative distributed system efficiently in context of constrained communication and computational resources along with high demands of medical applications.

The structure of the paper is as follows. First, the collaborative teleradiology system architecture requirements are presented and discussed in details. Features of this system are then compared to the web centric approach. Particular attention has been put to network QoS requirements, which result from different large data set sharing models. Next, synchronization techniques, which are essential to any collaborative distributed system, are presented. Finally, an example collaborative teleradiology application called TeleDICOM is presented. The system is under development and will be introduced to a medical practice in Krakow Center of Telemedicine. The paper ends with conclusions.

\section{Web-Centric Approach vs. Collaborative Systems}

Collaborative teleradiology could be understood as a new radiology medical datasharing pattern for telemedicine. To understand better what collaborative work means is it necessary to refer to the definition of collaborative computing systems [6] and compare it to a web centric approach.

In the context of teleradiology, collaborative computing means application sharing [7] that allows:

- taking an existing single user application and making it shareable,

- broadcasting graphics, mouse movements, and edits to all participants,

- usage of telepointer and "Master" pointer,

- integration with audio, video and text chat connections.

A single-user application that could be made shareable over a network could be, for instance, a DICOM images viewer commonly used in any digital radiology system. This simple concept requires construction of highly interactive, collaborative environment, which should provide abstraction of a session (i.e., a group of objects associated with some common communication pattern), and support full-duplex multi-point communication among arbitrary number of connected application entities.

The primary functionality provided by the collaborative teleradiology application is to send digital images to all of the participants within a communication session. Each of the images is represented as a shared byte array, so that any modification made by a session member is automatically reported to all of the session members. This ability should be enhanced with synchronization facilities, e.g. possibility to ensure mutually exclusive modification of the shared images. In teleradiology application, such a modification of image could be understood as e.g.: zooming, making adjustments to Hounsfield scale window, marking regions of interest etc. 
The idea of a collaborative teleradiology application is depicted in Fig.1. It is possible to identify four basic steps of its activity:

1. The same radiology image is retrieved from a database and distributed over the teleradiogy session members' computers.

2. The image is displayed on every computer screen of teleradiology session members when teleradiogy session is started.

3. All session members are notified about modification of the image made by one of them, so that the state of consulted data is synchronised.

4. Results of the teleradiology session (e.g. its recording) are stored back in a database to be accessed when necessary.

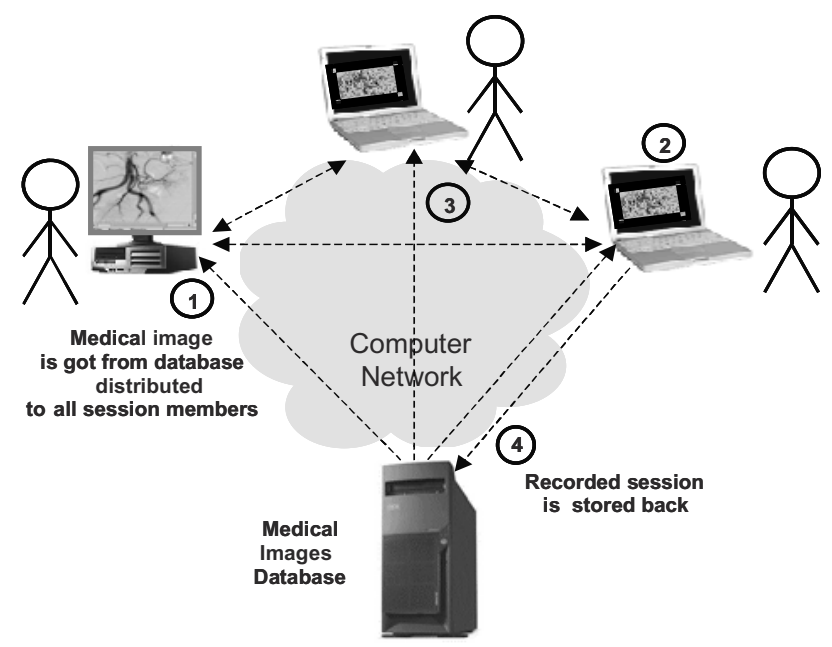

Fig. 1. Collaborative teleradiology application model

Such a scenario, with possibility of audio and video communication between the session members, would follow our definition of collaborative teleradiology.

Collaborative teleradiology could be easy compared with the web centric approach. Basic steps of the web-centric teleradiology application are depicted in Fig.2 and could be described as follows:

1. A selected radiology image is fetched from a database by dedicated software installed on a web server and sent to a user's web browser.

2. A radiology expert analyses the image.

3. A diagnosis in form of comments and added annotations is posted back to web server and stored in the database.

4. The diagnosis is read by other medical personnel when needed.

The basic difference between the collaborative and web centric approaches is that the latter does not provide interactivity, and comments could not be exchanged on-line between medical experts. This makes the analysis difficult and less efficient. 
The presented general model of collaborative teleradiology applications could be further refined by defining many simple subcases. Such subcases would be taking into account QoS constrains and scalability of the system. We will continue this considerations in the following sections.

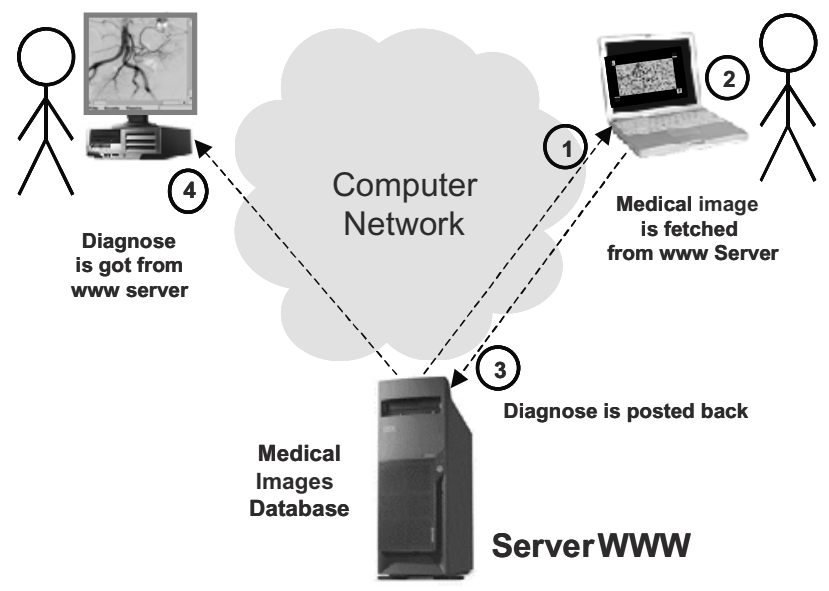

Fig. 2. Web-centric teleradiology

\section{Models of Data Sharing}

Radiology images are commonly represented according to DICOM standard [8]. The size of files containing radiology data stored in that format ranges from a few megabytes up to many tenths of megabytes. This is why high-speed broadband computer networks are required for fast on-line access to DICOM images. When using ISDN lines, the transfer of a DICOM file could take from a few minutes up to half an hour or even more. This is a real constraint of teleradiology application in the area where modern and expensive network infrastructure is not present. In that sense teleradiology application is network QoS (Quality of Service) dependent.

The solution to this problem is to reduce the amount of data transmitted during teleradiology sessions. For that purpose in Web centric applications the DICOM images are annotated by smaller in size JPEG pictures used for preview of radiology data. Doctors are using JPEG pictures to select interesting DICOM files, which have to be transmitted from a database to a DICOM viewer application or a web browser with DICOM viewer plug-in present on client machine. Unfortunately, the transmission of the selected DICOM files still takes a lot of time when it is performed over slow computer network. The discussed technique is used in commercially available radiology software e.g. ACOMWEB [9].

The network throughput constraint is even more serious for collaborative teleradiology applications, when on-line DICOM files transmission is assumed. The solution is to separate the collaborative teleradiology session into two phases: 
1. Distribution of DICOM images to the computers of the teleradiology session members. This operation could be made off-line, e.g. at night.

2. Collaborative processing of DICOM images, which concerns the points 2,3 and 4 of collective teleradiology model disscused in Section 2.

Separation of these two phases makes sense, because the second phase is not as data transfer intensive as the first one. Transmission of synchronization and control information needed to implement collaborative processing of DICOM images generates data stream that could be transmitted even over ISDN lines.

The complexity of collaborative teleradiology applications is substantially dependent on the number of session members. From that point of view, two scenarios could be identified:

1. Dual-party teleradiology session, when only one radiology expert in a reference center and one doctor in a remote hospital are involved in collaboration.

2. Multi-party teleradiology session, with more than two members involved.

The effectiveness of the implementation of a multi-party teleradiology session depends on multicast communication support provided by the network infrastructure. The availability of reliable multicast communication has a great influence on the scalability of implementation of synchronization mechanisms and will be elaborated further in the following section.

\section{Synchronisation of States of Multiple Distributed Teleconsultation Applications}

One of the features that a user would expect from a teleconsultation application is synchronization of the state of remote machines. From a user point of view, the applications must ensure that any actions performed by one of cooperating parties will be noticed immediately by all the others. To make the matter more complex, the users of the applications do not frequently have the same privileges. As an example, consider a scenario when a real life teleconsultation is observed by many remotely taught students, who definitely would not have rights to modify the diagnosis. In such case, the teleconsultation system would need to implement some access restrictions ${ }^{1}$.

Another issue, which seems to be important, is permitting the priviledged users to gain a lock on selected objects. In such case, the network of collected applications should for a while perform a ,one-to-many" transmission scenario, with one source of messages and many sinks ${ }^{2}$. Therefore, the system needs a powerful middleware platform to send messages between its entities.

In today's highly distributed systems, two general forms of message-oriented middleware are commonly found: point-to-point and publish-subscribe. The first, often called message queuing, is used to move data between its source and destination, where the messages wait in a queue for application to pick them up. The

\footnotetext{
${ }^{1}$ Another important issue is the confidentiality of consulted patient's personal data.

${ }^{2}$ Moreover, the system should provide a fault recovery procedure in case when a client holding locks fails.
} 
second kind is generally used when a data item needs to be sent to multiple clients. It can occur either if there is one or more sources of data.

The CORBA architecture, which is an industry standard, defines two ways of decoupled, event-driven communication, realised by Event and Notification Services, respectively. The OMG Event Service supports asynchronous exchange of event messages between objects. It introduces the following concepts:

- event channels, which function as brokers of event messages,

- event suppliers, which supply event messages, and

- event consumers, consuming i.e. receiving event messages.

The OMG Notification Service extends OMG Event Service by supporting a few important additional capabilities, e.g. filtering events the client wants to receive and possibility to specify various quality of service properties.

The Java Shared Data Toolkit software allows developers to add collaboration features to applets and applications written in the Java programming language. By implementing a multipoint data delivery service the toolkit can aid implementing network-centric applications, such as shared whiteboards or chat environments. The area of usage the JSDT includes also networking multimedia applications.

The Java Message Service (JMS) is a standardised set of programming interfaces to middleware. The middleware packages differ not only in their implementation details, but also in their interfaces. Since numerous middleware vendors include support for JMS in their products ${ }^{3}$, it has become a way to escape from the vendor „lock-in" that many distributed systems are suffering from. In a way, it bridges the communication between wide variety of different software components (both servers and clients), e.g. firewalls, mail servers, application servers and so forth, which are standard entities for most of today's distributed systems. JMS supports both message queuing and publish-subscribe communication patterns.

In general, JMS could be useful for communication between different, loosely coupled system entities that make use of asynchronous communication. JMS supports one-to-one, one-to-many and many-to-many configurations.

The medical teleconsultation systems described later in this paper fall into the first and third category, respectively, so it seems that they could be served by JMS well. However, a teleconsultation application needs to be highly interactive. This feature is expected from the system even in the case of low-bandwidth connection between peripheral and referential hospital. Since that, one of the most important things to do during the design and implementation phases is to reduce any transmission overhead.

\section{TeleDICOM System}

The applications used in the Krakow Centre of Telemedicine formerly gave only the possibility to send digitised patients' data to the consulting centre and view it independently with no interaction. The only connection between the both sides was a

${ }^{3}$ This popularity of JMS is partly a result of a requirement of JMS compliance, which needs to be fulfilled by any software component in order to get a J2EE certification. 
voice (or sometimes also video) channel, often realised via separate technical solutions (e.g. telephone line). The experience drawn from the TeleNegatoscope application allowed designing a more advanced environment.

TeleDICOM is going to offer many more advanced features introduced by the collaborative teleradiology paradigm. The most important of them is that it will operate on DICOM images offering fully diagnostic quality and precise textual description. DICOM format is de facto standard in the modern medical world; the images are very often created directly by medical equipment, e.g. a computer topography. The idea of the TeleDICOM environment originates from whiteboardlike applications where many participants share the same area making changes in its appearance or just observing modifications performed by the others. In TeleDICOM, a DICOM image delivered to all participants early enough will form the background. Operations performed on the image by one of the participants will be propagated to all the others. The available operations are supposed to be helpful during a consultation allowing users e.g. to mark fragments of the image (by placing different shapes there). The operations will also include actions typical for DICOM data, e.g. modifying the Hounsfield scale window parameters, zooming the image or running animation mode if the image is a multi-sliced one (e.g. coronarography examination).

The application has also an educational role allowing medicine students to participate in real consultations.

One of the most important design issues was how to organise flow of data in a multiparty scenario effectively. Taking into consideration the problems with multicast routing and reliable multicast, the decision was to use a centralised star topology with so called rendez-vous points to which all participants would direct their requests, and which would notify all participants about actions performed by the others. The whole communication is accomplished by using unicast transmission. Such a rendez-vous point, called session server, can be integrated into one of the applications participating in the consultation, or be run independently. The former solution is preferred in small, dual-party sessions and the latter in the multi-party ones. The logical placement of the session server in such case should be determined taking into account the location of all participants and aiming at ensuring them nearly equal distance to the central node of the star architecture in terms of available bandwidth and transmission delay. The concept of the session server in a session containing several participants is depicted in Fig. 3.

Security of medical information cannot be forgotten and the authors of TeleDICOM kept this in mind. All centres using the application have to get certificates used for authentication. The medical data (DICOM files and diagnoses) are encrypted by using SSL while being sent. The tests performed by authors proved that encryption of control data during consultation phase, severely influences its interactivity making it suffer from unacceptable delays, unless very fast computers are used. It must be however emphasised that with no knowledge about medical data itself, information intercepted by an eavesdropper is practically useless. In cases when a very strong security is required transmission can be led via Virtual Private Networks (VPNs) with fast encryption mechanisms provided on lower layers. 


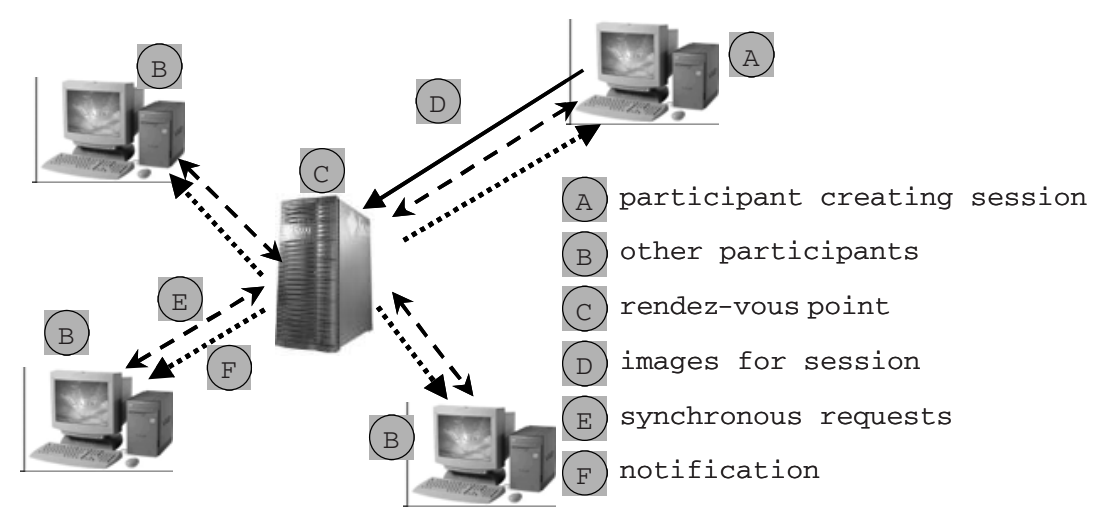

Fig. 3. The concept of rendez-vous point (session server) in multiuser session

\section{Conclusions}

The collaborative teleradiology opens a new, very challenging, field of multidisciplinary research in the area of telemedicine. The progress in this domain is possible due to the application open standards in distributed computing, which create a solid foundation for not only experimental, but also commercial, fully distributed, applications. Collaborative telemedicine put applied distributed software engineering in the context of very demanding applications in terms of required QoS, security and synchronised visualization techniques.

There is also open area for ergonomical and sociological studies to understand the requirements of the user interface design for group communication and on-line data sharing better. This research needs construction and deployment of a whole family of software tools supporting the proposed collaborative model and collecting experiences from the pilot systems introduced to the clinical practice.

\section{References}

1. NetRAAD system, University Health Care Company, http://www.uhc.com.pl

2. Web PACS, Universal PACS Inc., http://www.unipacs.com/webpacs.html

3. CORBAservices: Common Objects Services Specification, OMG, 1998

4. Remote Method Invocation Specification, Sun Microsystems, 1997

5. Enterprise Java Beans Specification, version 2.1, Sun Microsystems, 2002

6. Collaborative computing, Collab Worx Inc., http://www.collabworx.com/Support/resources

7. Java Shared Data Toolkit, Sun Microsystems, http://java.sun.com/products/java-media/jsdt/

8. The DICOM Standard, National Electrical Manufacturers Association.

9. Acom.WEB, Universitätsklinikum Essen, http://www.uni-essen.de/cardio/acomweb/ acomweb_info.html 